\title{
Early Ophthalmological Manifestations of Crouzon Syndrome: About a Case
}

\author{
Nidain Maneh1 ${ }^{*}$ Meba Banla1, Nadiedjoa Kokou Douti², Komi Yayehd ${ }^{3}$, Sophie Arsene ${ }^{4}$, \\ Kokou Vonor ${ }^{1}$, Kassoula Batomaguela Nonon Saa ${ }^{1}$, Koffi Didier Ayena1, Komi Patrice Balo ${ }^{1}$ \\ ${ }^{1}$ Eye Department chu Campus, University of Lomé, Lomé, Togo \\ ${ }^{2}$ Department of Paediatric chu Campus, University of Lomé, Lomé, Togo \\ ${ }^{3}$ Heart Department chu Campus, Lomé, Togo \\ ${ }^{4}$ Eye Department chu, Tours, France \\ Email: "manehnid@hotmail.fr
}

Received 1 August 2014; revised 25 September 2014; accepted 26 October 2014

Copyright (C) 2014 by authors and OALib.

This work is licensed under the Creative Commons Attribution International License (CC BY).

http://creativecommons.org/licenses/by/4.0/

(c) (i) Open Access

\begin{abstract}
We report the case of a major neonatal form of Crouzon syndrome of ophthalmic finding in a newborn of 21 days of age with no background of consanguinity. The clinical manifestations were marked by signs of Crouzon syndrome associated with dyspnea. The skull radiograph showed a decline of maxillary and closing sutures. The brain scan was marked by a bilateral fronto-parietal hypotrophy with agnenesis of the left temporal. She had received a lateral tarsorrhaphy in prevention to keratopathy of exposure. We noted a substantive consideration of normal eye and no recurrence throttle of eyeball 6 months after tarsorrhaphy. The major forms of Crouzon syndrome is a source of complications including exposure keratopathy and optic atrophy requiring ophthalmological caring in awaiting for a craniofacial surgery.
\end{abstract}

\section{Keywords}

Craniofacial Dysostosis

Subject Areas: Ophthalmology, Pathology, Public Health

\section{Introduction}

Le syndrome de Crouzon est une sténose cranio-faciale caractérisée par la synostose précoce de la suture coronale parfois des sutures lambdoïdes et sagittales [1]. Cette dysmorphie faciale comporte une hypoplasie maxillaire, un exorbitisme, un hypertélorisme [2]. Maladie rare, elle est évolutive et apparaît cliniquement le plus souvent après l'âge de 2 ans en dehors des formes majeures à révélation néonatale.

"Corresponding author. 


\section{Observation}

Nous rapportons le cas d'un nouveau-né de sexe féminin de 21 jours de vie extra-utérine adressé par le service de pédiatrie pour une exophtalmie bilatérale.

Ses antécédents personnels révèlent qu'elle est issue d'une grossesse normale, et d'un accouchement par voie basse sans complications en dehors d'une dyspnée.

Dernière d'une fratrie de trois enfants, son frère et sa sœur sont vivants et bien portants. Son père et sa mère âgés respectivement de 41 ans et 35 ans ne présentent aucune anomalie physique et sont non consanguins.

\section{Examen Clinique (Figure 1)}

A l'examen ophtalmologique il a été noté:

- un exorbitisme bilatéral plus prononcé à gauche;

- une lagophtalmie non permanente après l'étranglement récidivant du globe par les paupières;

- un hypertélorisme et un strabisme divergent;

- un examen normal du fond d’œil sous anesthésie générale.

Les anomalies de la tête et de la face sont marquées par un retrognatisme maxillaire, une macroglossie avec une lippe de la lèvre inférieure épaisse et un nez crochu en bec de perroquet. L'auscultation cardiaque a noté un souffle systolique en rayon de roue. On n’a pas constaté de syndactylie des mains ni des pieds.

\section{Examens Paracliniques (Figure 2 et Figure 3)}

La radiographie standard du crâne a précisé la synostose crânienne, un recul du maxillaire supérieur et un téléorbitisme.

La tomodensitométrie cérébrale a relevé une exophtalmie, une hypotrophie fronto-pariétale bilatérale avec agénésie temporale gauche associée à une asymétrie hémisphérique.

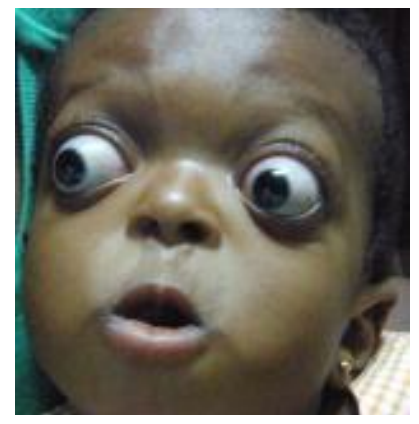

Figure 1. Retrognatisme, nez crochu, hypertélérisme et exorbitisme important.

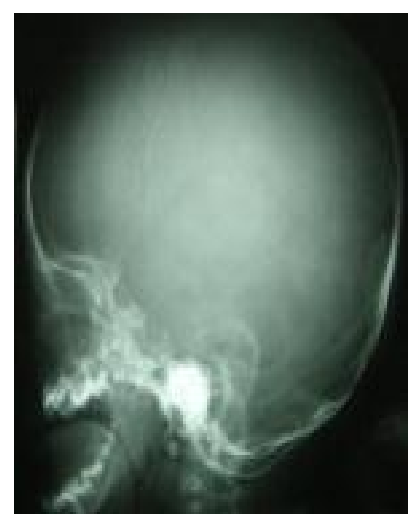

Figure 2. Retrognatisme, nez crochu, hypertélérisme et exorbitisme important. 


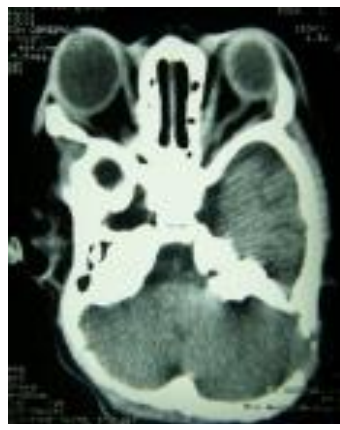

Figure 3. Exophtalmie, agénésie temporale gauche et Hypotrophie fronto-pariétale bilatérale.

L'échographie doppler cardiaque a retrouvé une communication inter-auriculaire (CIA) faite de deux trous; un shunt gauche-droit avec un retentissement hémodynamique modéré. Le caryotype n’a pas pu être réalisé après le refus des parents.

\section{Traitement}

En attente d'un traitement chirurgical dans un centre mieux équipé, une tarsorraphie externe avait été réalisée pour réduire le risque d'exposition cornéenne et d'étranglement du globe oculaire par les paupières.

Durant les trois mois post opératoires on a noté une disparition des épisodes d'extériorisation du globe oculaire avec une cornée restée transparente et un fond d'œil toujours normal.

\section{Commentaire}

Les signes cliniques sont fortement évocateurs du syndrome de Crouzon décrit la première fois en 1912 par Louis Edouard Octave Crouzon [2]. Il existe des formes précoces congénitales comme dans notre cas, dans lesquelles l'hypoplasie maxillaire est majeure et responsable de difficultés respiratoires et d'exorbitisme. Des complications cardiaques et viscérales sont également décrites dans ce syndrome [3].

L'origine génétique de la maladie est actuellement admise avec un mode de transmission héréditaire autosomique dominant à expressivité variable [4]. Notre recherche étiologique a souffert du manque du caryotype avec l'absence du consentement des parents, du fait de leur préjugé sur la maladie, qui serait liée selon eux à une malédiction. Néanmoins l'absence de consanguinité des parents et d'antécédent familial chez notre patiente font penser à un cas sporadique également décrit dans la maladie.

Le risque d'hypertension intracrânienne par inadéquation entre le développement cérébral et celui du crâne ralentit par la synostose, est particulièrement important surtout dans cette forme congénitale à révélation néonatale. A ce sujet, certains auteurs rapportent que l'incidence de l'atteinte du nerf optique dans ces anomalies cranio-faciales varie de 54 à 100\% [5] [6].

Dans cette forme majeure, l' exposition cornéenne est importante avec le risque de kératopathie d'exposition [7]. La tarsorraphie externe dont a bénéficié notre patiente a permis d'éviter cette complication. Le traitement du syndrome de Crouzon est bien codifié et actuellement dominé par l'ostéotomie avec un avancement fronto-facial en monobloc [8], afin d'éviter les complications oculaires, cérébrales et esthétiques. Toutefois cette chirurgie doit être réalisée par une équipe pluridisciplinaire expérimentée dans un centre hautement spécialisé [9].

\section{Conclusion}

Les formes majeures à révélation néonatale du syndrome de Crouzon sont source de complications notamment oculaires et cérébrales. L'éviction des complications cornéennes en attente d'une chirurgie crânio-faciale est essentielle à réaliser pour préserver la vision et elle consiste à la réalisation d'une tarsorraphie externe.

\section{Remerciements}

Nous remercions particulièrement les parents de la patiente pour avoir marqué leur accord pour cette étude de cas. 


\section{References}

[1] Tessier, P., Rougier, J. and Wolfe, S.A. (1981) The Craniofaciostenosis (CFS): The Crouzon and Apert Disease, the Plagiocephalies. In: Masson, Ed., Plastic Surgery of the Orbits and Eyelids, New York, 200-223.

[2] Crouzon, O. (1912) Dysostose crânio-faciale héréditaire. Bulletins et Mémoires de la Société Médicale des Hôpitaux de Paris, 33, 545-555.

[3] Denis, D., Conrath, J. and Gentori, L. (1997) Exophtalmie, astigmatisme et strabisme dans les crânio-facio-sténoses: Syndrome d'Apert et syndrome de Crouzon. Ophtalmologie, 11, 28-33.

[4] Crouzon, O. (1915) Une nouvelle famille atteinte de dysostose cranio-faciale héréditaire. Archives de Médecine des Enfants (Paris), 18, 540-543.

[5] Miller, M. and Pruzansky, S. (1980) Cranio-Facial Anomalies. Principles and Practice of Ophthalmology. Saunders, Philadelphia, 3, 2367.

[6] Friedenwald, H. (1901) On Optic Nerve Atrophy Associated with Cranial Deformity. Arch Ophthalmology, 30, 405413.

[7] Chana, H.S. and Klauss, V. (1988) Crouzon’s Craniofacial Dysostosis in Kenya. British Journal of Ophthalmology, 72, 196-197. http://dx.doi.org/10.1136/bjo.72.3.196

[8] Guichard, B., Davrou, J., Neiva, C. and Devauchelle, B. (2013) Midface Osteotomies Lines: Evolution by Paul Tessier, the Second Tessier Classification. Journal of Craniomaxillofacial Surgery, 41, 504-515. http://dx.doi.org/10.1016/j.jcms.2013.04.003

[9] David, D.J. (2003) Advances in the Management of the Craniosynostosis. ANZ Journal of Surgery, 73, 949-957. http://dx.doi.org/10.1046/j.1445-2197.2003.02825.x 